\title{
Teste Rápido para Detecção da Infecção pelo HIV-1 em Gestantes
}

\author{
Rapid Test to Detect HIV-1 Infection among Pregnant Women \\ Geraldo Duarte, Carla Vitola Gonçalves, Alessandra Cristina Marcolin, Marina Carvalho Paschoini \\ Silvana Maria Quintana, Marisa M. Mussi-Pinhata
}

\begin{abstract}
RESUMO
Objetivos: avaliar os resultados do teste de diagnóstico rápido da infecção pelo HIV-1 disponibilizado pelo Ministério da Saúde, para identificação de gestantes contaminadas por este virus.

Métodos: avaliação prospectiva de 443 gestantes sem teste sorológico para HIV no pré-natal, atendidas no Departamento de Ginecologia e Obstetrícia da Faculdade de Medicina de Ribeirão Preto-Universidade de São Paulo (HCFMRP-USP), entre fevereiro e junho de 2000. As amostras destas pacientes foram submetidas ao teste rápido imunocromatográfico, sendo comparadas com ELISA e confirmadas pela aglutinação.

Resultados: dentre as 443 gestantes submetidas ao teste rápido (20,1\% dos partos no periodo), 16 apresentaram resultados positivos (3,6\%). Nenhuma amostra negativa pelo teste rápido foi positiva pelo ELISA. Entretanto, das 16 amostras positivas pelo teste rápido, duas foram negativas pelos testes confirmatórios. Logo, a sensibilidade do teste rápido foi de $100,0 \%$, especificidade $99,5 \%$, valor preditivo positivo $87,5 \%$ e valor preditivo negativo $100,0 \%$.

Conclusões: os resultados obtidos na avaliação do teste para o diagnóstico rápido da infecção pelo HIV-1 em gestantes revelaram sensibilidade, especificidade e valores preditivos que o credenciam como recurso extremamente importante na indicação de medidas que reduzem a transmissão perinatal desse vírus.
\end{abstract}

PALAVRAS-CHAVE: AIDS. Transmissão vertical. Pré-natal.

\section{Introdução}

Desde o seu reconhecimento como entidade clínica distinta em 1981, a sindrome da imunodeficiência adquirida (AIDS) vem impondo contínuos desafios aos obstetras e neonatologistas de todo o mundo. No singular período de gravidez, os cuidados ininterruptos desses profissionais extrapolam os aspectos médicos da síndrome na tentativa de reduzir tanto a morbidade materna naturalmente ligada ao processo gestacional como a transmissão vertical (TV) do vírus responsável

Setor de Moléstias Infecto-Contagiosas em Ginecologia e Obstetrícia do Departamento de Ginecologia e Obstetricia da Faculdade de Medicina de Ribeirão Preto - Universidade de São Paulo

Correspondência:

Geraldo Duarte

Hospital das Clínicas da Faculdade de Medicina de Ribeirão Preto - USP

Avenida Bandeirantes, 3900

14049-900 - Ribeirão Preto - SP

Fone: (16) 602-2588

e-mail: gduarte@fmrp.usp.br por esta sindrome ${ }^{1}$.

A AIDS é causada pelo vírus da imunodeficiência humana (HIV), um retrovirus da família Lentivirus, isolado na França por BarréSinoussi et al. ${ }^{2}$. Já foram relatados dois tipos diferentes deste vírus, conhecidos como HIV tipo 1 (HIV-1) e HIV tipo 2 (HIV-2) ${ }^{2}$. No Brasil, o tipo mais importante é o HIV-1.

Avaliando a evolução cronológica das taxas de prevalência de AIDS entre as mulheres, verifica-se que os números não são favoráveis a este segmento populacional. No início da epidemia a proporção de casos entre homens/mulheres no Brasil era de 40/1, chegando a 2/ 1 em 1999. Visto que o incremento verificou-se entre as mulheres em idade reprodutiva, é lógico que ocorra um aumento do número de casos de TV desse vírus, responsável por $95 \%$ dos casos de AIDS pediátrica ${ }^{3}$. Este dados reforçam a necessidade de uma ação diferenciada entre as mulheres, notadamente entre aquelas com possibilidades de engravida$\mathrm{rem}^{4}$. 
O HIV pode ser transmitido de uma mulher infectada para seu feto ou recém-nascido durante a gravidez, trabalho de parto, parto e durante o período puerperal pela amamentação. Embora a transmissão vertical possa ocorrer desde a oitava semana de gestação ${ }^{5}$, a utilização das técnicas de replicação dos ácidos nucléicos possibilitou demonstrar que pelo menos metade dos casos de infecção fetal acontecem imediatamente antes ou durante o parto ${ }^{6,7}$. A amamentação pode aumentar a taxa de transmissão em até $20 \%{ }^{8,9}$. De forma geral, considerando as várias partes do mundo, a transmissão vertical apresenta taxas que variam de 13 a $40 \%{ }^{10}$. Fica claro, portanto, que o final da gestação, o trabalho de parto e a amamentação são períodos críticos quando se fala em TV do HIV-1, cabendo priorizar as medidas que controlam essa forma de disseminação viral nestes períodos.

Connor et al. ${ }^{11}$ constataram que o uso do AZT em mulheres com doenças assintomáticas e sem tratamento com anti-retrovirais durante a gestação reduz o risco de TV em 67,5\%. Este regime foi recomendado pelos órgãos oficiais de prevenção dos Estados Unidos da América naquele mesmo ano. Entretanto, no Brasil, apesar do regime antiretroviral profilático ser oferecido gratuitamente pelo Ministério da Saúde, ainda existem sérias dificuldades na identificação das gestantes infectadas por este vírus, privando-as do acesso às medidas profiláticas da TV.

Com a descoberta da possibilidade de reduzir a transmissão vertical tornou-se imprescindivel a descoberta de métodos diagnósticos precisos, acessiveis em termo de custos e tecnologia. Apesar do avanço que representa, o ELISA não permite resultados rápidos, limitando seu uso na identificação de pacientes em situação de urgência diagnóstica, como a gestante em trabalho de parto. Para esta finalidade foram desenvolvidos os "testes rápidos", mais simples, rápidos e de baixo custo, permitindo a identificação das gestantes portadoras do vírus e instituição de medidas que reduzem a transmissão vertical desse microrganismo.

O objetivo deste trabalho foi avaliar os resultados do teste de diagnóstico rápido da infecção pelo HIV-1 oferecido pelo Ministério da Saúde do Brasil, para a identificação de gestantes portadoras dessa infecção e que não tinham exame sorológico prévio.

\section{Pacientes e Métodos}

Foi feita uma avaliação prospectiva de 443 gestantes, sem teste sorológico de anticorpos anti-
HIV no pré-natal, atendidas no Departamento de Ginecologia e Obstetrícia da Faculdade de Medicina de Ribeirão Preto-Universidade de São Paulo (HCFMRP-USP), entre fevereiro e junho de 2000. Após aconselhamento e anuência das pacientes em participarem do estudo, as amostras destas pacientes foram submetidas ao teste rápido (Determine ${ }^{\mathrm{TM}}$ - Abbott Laboratórios do Brasil). Dentre as informações do aconselhamento, destacou-se o fato de o teste não ter caráter diagnóstico definitivo, apenas orientador para uso de AZT profilático.

De todas as pacientes foram coletadas amostras sangüineas que foram submetidas ao teste rápido e ao teste ELISA. As amostras com resultados ELISA positivos eram confirmadas pelo teste da aglutinação ou imunofluorescência. O princípio do teste rápido em pauta é imunocromatográfico, detectando qualitativamente anticorpos contra o HIV-1/2. Considerou-se como resultado positivo o aparecimento de duas barras vermelhas (uma no espaço correspondente ao controle e outra no espaço correspondente à amostra da paciente). Como resultado negativo a barra vermelha aparecia apenas no controle e o resultado foi considerado inválido, quando não se observou nenhuma barra, nem mesmo no controle. Os testes ELISA e os testes confirmatórios foram realizados no laboratório de sorologia do HCFMRP-USP, seguindo as normas dos fabricantes.

As gestantes com teste rápido positivo para infecção pelo HIV-1 foram admitidas no protocolo do Setor de Moléstias Infecto-Contagiosas em Ginecologia e Obstetrícia do HCFMRP-USP que, nestes casos, orienta o uso de AZT materno durante o trabalho de parto e no neonato, suspendendo-se a amamentação. Nos casos confirmados de infecção pelo HIV-1, após a alta hospitalar as pacientes foram encaminhadas para seguimento no setor especializado. Os recém-nascidos dessa casuística estão em seguimento no Ambulatório de Infectologia Pediátrica do HCFMRP-USP. No caso de pacientes cujas amostras foram positivas pelo teste rápido, mas os testes confirmatórios foram negativos, o uso de AZT nos recém-nascidos foi suspenso.

Para avaliar o desempenho do teste rápido para o diagnóstico da infecção pelo HIV-1 em parturientes, foram calculadas a sensibilidade, especificidade e os valores preditivos negativo e positivo.

Resultados

Das 443 gestantes submetidas ao teste rápido para o diagnóstico da infecção pelo HIV-1 
( $20,1 \%$ dos partos ocorridos no período), observouse que 16 pacientes apresentaram resultados positivos para anticorpo anti-HIV $(3,6 \%)$ e 427 apresentaram resultados negativos para anticorpo antiHIV $(96,4 \%)$, conforme mostram os dados da Tabela 1. O teste ELISA e os testes confirmatórios (aglutinação e imunofluorescência) confirmaram positividade em 14 das 16 amostras que apresentaram resultados positivos para anticorpos antiHIV com teste rápido (duas amostras foram falsopositivas). Dentre as 427 amostras negativas para anticorpos anti-HIV ao teste rápido, nenhuma apresentou resultado positivo ao ELISA e aos testes confirmatórios. Sendo assim, a sensibilidade do teste rápido foi de $100,0 \%$, a especificidade $99,5 \%$, o valor preditivo positivo $87,5 \%$ e o valor preditivo negativo $100,0 \%$.

Tabela 1 - Dados comparativos entre 443 gestantes submetidas ao teste rápido para diagnóstico da infecção pelo HIV e aos testes ELISA e aglutinação padronizados no HCFMRP-USP.

\begin{tabular}{lrrrrrr}
\hline Teste Rápido & \multicolumn{3}{c}{ Elisa e Testes Confirmatórios } & \multicolumn{2}{c}{ Total } \\
& \multicolumn{2}{c}{ Positivo } & \multicolumn{2}{c}{ Negativo } & & \\
& $\mathbf{n}$ & $\%$ & \multicolumn{1}{c}{$\mathbf{n}$} & \multicolumn{1}{c}{$\%$} & \multicolumn{1}{c}{$\mathbf{n}$} & \multicolumn{1}{c}{$\%$} \\
\hline Positivo & 14 & 3,2 & 2 & 0,4 & 16 & 3,6 \\
Negativo & 0 & 0 & 427 & 96,4 & 427 & 96,4 \\
Total & 14 & 3,2 & 429 & 96,8 & 443 & 100,0 \\
\hline
\end{tabular}

Nota: sensibilidade: $100,0 \%$; especificidade: $99,5 \%$; valor preditivo positivo: $87,5 \%$; valor preditivo negativo: $100,0 \%$.

\section{Discussão}

Após a entrada do HIV-1 no organismo ocorre o seu reconhecimento pelo sistema imunológico, provocando resposta humoral, tornando positiva a presença de anticorpos contra o virus, em média, entre quatro e oito semanas. $\mathrm{Na}$ tentativa de facilitar o diagnóstico do HIV-1, pesquisadores desenvolveram métodos laboratoriais inovadores mas que já vinham sendo investigados desde o início da década de 80. Porém, somente em 1985, testes de detecção de anticorpos antiHIV tornaram-se disponiveis para uso rotineiro, como os ensaios imunoenzimáticos (ELISA), testes de elevada sensibilidade, mas com especificidade que demandava testes confirmatórios. Para o diagnóstico da infecção pelo HIV-1, o Ministério da Saúde do Brasil preconiza que seja realizado o ELISA (de alta sensibilidade), como método de triagem, e um outro método como teste confirmatório (aglutinação de partículas de látex, imunofluorescência indireta e Western-blot) ${ }^{12,13}$.

Com a evolução tecnológica, os testes ELISA disponiveis no mercado na atualidade apresentam também boa especificidade. Apesar da possibilidade de automação, os testes ELISA para diagnóstico da infecção HIV ainda não conseguiram atender a questão referente à urgência diagnóstica, visto que seus resultados demoram aproximadamente duas horas para serem expedidos, havendo limitações econômicas e técnicas para a execução do teste em uma amostra isoladamente.

Em face dos excelentes resultados obtidos com a utilização do AZT (pré-natal, durante o trabalho de parto e no período neonatal) reduzindo o risco de TV do HIV-1 em 67,5\%, este regime foi recomendado pelos órgãos oficiais de prevenção dos Estados Unidos da América em 1994 ${ }^{11}$. Entretanto, o alto custo e a complexidade desse regime profilático não permitiu sua implantação em vários dos países em desenvolvimento. Em 1996, o Ministério Público da Tailândia, em colaboração com os Centers for Disease Control and Prevention (CDC), elaborou um novo estudo randomizado, duplo cego com controle placebo, observando redução de $51 \%$ de transmissão vertical com o uso de AZT via oral a partir de 36 semanas de gestação e no período intraparto, sem administração da droga aos recém-nascidos no período neonatal ${ }^{14}$. Um esquema alternativo foi sugerido com o uso de nevirapina antes do parto e no recém-nascido, apresentando uma redução de $47 \%$ em relação aos resultados obtidos com o esquema completo do $\mathrm{AZT}^{15}$. Entretanto, no Brasil, apesar de o regime anti-retroviral profilático estar disponibilizado gratuitamente pelo Ministério da Saúde, ainda existem sérias dificuldades na identificação das gestantes infectadas por este virus, privando-as do acesso às medidas profiláticas da TV.

Os avanços no entendimento da transmissão vertical do HIV-1 indicam que aproximadamente dois terços da transmissão vertical desse vírus ocorrem no periodo periparto e durante a amamentação natural. Entretanto, aproximadamente um quinto das parturientes chegam às maternidades de nossa região sem teste prévio e, portanto, sem acesso aos beneficios do uso do AZT intraparto e neonatal e às orientações para suspensão do aleitamento materno. Um teste de diagnóstico rápido da infecção pelo HIV-1 no momento da internação destas pacientes poderia resolver este problema.

$\mathrm{O}$ primeiro teste rápido realizado para diagnóstico da infecção HIV-1 utilizou saliva para detecção de anticorpos contra esse vírus, sendo usado até hoje em pesquisas, mostrando elevada sensibilidade e especificidade $(99,4 \%$ e $99,4 \%$, respectivamente) ${ }^{16}$. Seguiu-se a realização destes testes em sangue total, plasma, soro e urina. Entre os testes rápidos mais utilizados figura o Determineä, marca produzida Abbott Laboratórios do Brasil e dis- 
tribuído pelo Ministério da Saúde de nosso país. No estudo realizado por Kelen et al. ${ }^{17}$, em que foram avaliados 1.448 pacientes, o teste rápido em questão mostrou sensibilidade de $100 \%$ e especificidade de $98,9 \%$, com 5 resultados falso-positivos e nenhum falso-negativo. Um fato relevante observado neste trabalho foi a correlação entre tempo de obtenção do resultado do teste e a espera do paciente pelo mesmo. Dos pacientes submetidos ao ELISA, $55 \%$ abandonaram o estudo antes de saber o resultado, que apresenta tempo de execução em torno de $107 \pm 52$ min. Por outro lado, apenas $20 \%$ dos pacientes submetidos ao teste rápido abandonaram o estudo antes de sua conclusão, já que este teste apresenta um tempo de resolução em torno de $48 \pm 37 \mathrm{~min}^{17}$. O teste utilizado no presente trabalho possibilita leitura em 15 minutos, possibilitando agilizar uma série de medidas no sentido de administrar o AZT endovenoso para essas parturientes e iniciar o processo de aderência ao não aleitamento. Na presente casuística nenhuma paciente recusou a profilaxia com o antiretroviral.

Lien et al. ${ }^{18}$ compararam a performance de três kits de testes rápidos para HIV-1/2 aplicados em populações dos hospitais e clínicas do Vietnã. Segundo a Organização Mundial da Saúde (OMS), o teste utilizado no presente estudo apresentou melhor performance que os outros disponiveis no mercado, com menor taxa de resultados equivocados ou indeterminados ${ }^{18}$.

Palmer et al. ${ }^{19}$ participaram de um estudo multicêntrico que visava avaliar a sensibilidade e a especificidade do teste rápido imunocromatográfico. Os resultados encontrados foram de $100 \%$ tanto para sensibilidade como para especificidade, mostrando ser este um método diagnóstico de grande valor para identificação rápida de indivíduos portadores do HIV, especialmente em países em desenvolvimento com infra-estrutura laboratorial limitada ${ }^{19}$. Resultados semelhantes com relação à sensibilidade e especificidade deste teste também foram encontrados por Arai et al..$^{20}$, em pesquisa realizada na Tailândia. Os resultados das 443 amostras analisadas na presente casuística mostraram $100 \%$ de sensibilidade e $99,5 \%$ de especificidade, valores bem próximos àqueles descritos na literatura.

Várias pesquisas têm revelado o grande valor da detecção da infecção pelo vírus HIV em gestantes, por meio de testes rápidos, possibilitando o tratamento profilático da transmissão vertical com o uso de anti-retrovirais, reduzindo os custos com crianças contaminadas, principalmente em pacientes sem cuidados pré-natais. Para avaliar custobenefício do teste rápido na prevenção da transmissão vertical do HIV-1, associando-o ao tratamento com anti-retrovirais, Stringer e Rouse ${ }^{21}$ avaliaram três grupos de gestantes sem assistência pré-na- tal: um não submetido aos testes diagnósticos ou tratamento algum, outro submetido ao teste rápido e a tratamento com anti-retrovirais nos casos positivos e um terceiro que recebeu anti-retrovirais sem confirmação diagnóstica. Nos casos sem tratamento, ocorreram 1.275 casos de transmissão vertical por 100.000 gestações, com gastos em torno de 103.708 dólares por criança infectada. Com o uso do teste rápido seguido pela administração de zidovudina (AZT), preveniram-se 183 casos, economizando-se US\$57.997 por caso. Por outro lado, ao se tratar todas as pacientes, obteve-se prevenção de 46 casos por 100.000 gestações, mas o custo por caso aumentou para 342.068 dólares. Assim, a realização do teste rápido em gestantes sem pré-natal, seguido pela profilaxia com AZT dos casos soropositivos, parece ter excelente relação custobeneficio para o sistema de saúde ${ }^{21}$. Na última avaliação referente à TV no HCFMRP-USP, avaliando crianças que utilizaram AZT no período pós-natal e não receberam leite materno, verificou-se que a mesma ocorreu em 5,4\% delas ${ }^{22}$, representando uma redução de $73,2 \%$ nas taxas usuais de transmissão vertical do serviço (20,0\%). Fazendo analogia com as 14 parturientes e neonatos da presente casuística é possivel deduzir que pelo menos duas crianças deixaram de ser infectadas, representando uma economia de 115.994 dólares.

Em outra avaliação feita por Grobman e Garcia $^{23}$, evidenciou-se que na ausência de teste sorológico no pré-natal, o uso do teste rápido para detecção do HIV não só ajudou no diagnóstico mas também na profilaxia, prevenindo a transmissão vertical desse virus, com benefícios claros e indiscutiveis.

Em resumo, o teste rápido para diagnóstico da infecção pelo HIV-1 é uma excelente opção para uso em maternidades que atendem parturientes com elevada taxa da infecção pelo HIV-1 e baixa taxa de teste sorológico no pré-natal.

\section{SUMMARY}

Purpose: to evaluate the results of a rapid diagnostic test for HIV-1 infection made available by the Health Ministry for the identification of pregnant women contaminated by this virus.

Methods: we evaluated prospectively 443 pregnant women with no prenatal serologic anti-HIV test seen at the Department of Gynecology and Obstetrics of the Faculty of Medicine of Ribeirão Preto, University of São Paulo, from February to June, 2000. Samples from these patients were submitted to the rapid immunochromatographic test, which was compared with ELISA and submitted to a confirmatory agglutination test. 
Results: among the 443 pregnant women submitted to the rapid test (20.1\% of the deliveries performed during the study period), 16 showed positive results (3.6\%). No sample with a negative result by the rapid test was positive by ELISA. However, of the 16 samples that were positive by the rapid test, two were negative by the confirmatory tests. Thus, the rapid test showed $100.0 \%$ sensitivity, $99.5 \%$ specificity, $87.5 \%$ positive predictive value, and $100.0 \%$ negative predictive value.

Conclusions: the results obtained by evaluation of the test for a rapid diagnosis of HIV-1 infection in pregnant women revealed sensitivity, specificity and predictive values that qualify it as an extremely important resource for the indication of measures that will reduce perinatal transmission of this virus.

KEY WORDS: AIDS, vertical HIV-1 transmission. HIV, prenatal serodiagnosis

\section{Referências}

1. Duarte G. Síndrome da imunodeficiência adquirida tipo-1 e gravidez. In: Cunha SP, Duarte G, editores. Gestação de Alto Risco. $1^{a}$ ed. São Paulo: Medsi; 1998. p.227-46.

2. Barré-Sinoussi F, Cherman JC, Rey F, et al. Isolation of a T-lymphotropic retrovirus from a patient at risk for AIDS. Science 1983; 220:868-71.

3. Brasil. Ministério da Saúde. Transmissão vertical do HIV. Bol Epidemiol AIDS 1999; 12:5-57.

4. Bryson YJ. Perinatal HIV-1 transmission: recent advances and therapeutic interventions. AIDS 1996; 10 Suppl 3:S33-42.

5. Lewis SH, Reynolds-Kohler C, Fox HE, Nelson JA. HIV-1 in trophoblastic and villous Hofbauer cells, and haematological precursors in eight-week fetuses. Lancet 1990; 335:565-8.

6. Rogers MF, Ou C-Y, Rayfield M, et al. Use of the polymerase chain reaction for early detection of the proviral sequences of human immunodeficiency virus in infants born to seropositive mothers. N Engl J Med 1989; 320:1649-54.

7. Rouzioux C, Costagliola D, Burgard M, et al. Timing of mother-to-child HIV-1 transmission depends on maternal status. AIDS 1993; 7 Suppl 2:S49-52.

8. Bertolli J, St. Louis ME, Simonds RJ, et al. Estimating the timing of mother-to-child tranmission of human immunodeficiency virus in a breast-feeding population in Kinshasa, Zaire. J Infect Dis 1996; 174:722-6.

9. Ekpini ER, Wiktor SZ, Satten GA, et al. Late postnatal mother-to-child transmission of HIV in Abidjan, Cote d'Ivoire. Lancet 1997; 349:1054-9.

10.United States. Public Health Service. Recommendations for human immunodeficiency virus counseling and voluntary testing for pregnant women. Morb Mortal Wkly Rep 1995; 44:1-15.

11.Connor EM, Sperling RS, Gelber R, et al. Reduction of maternal-infant transmission of human immunodeficency virus type 1 with zidovudine treatment. N Engl J Med 1994; 331:1173-80.

12. Machado AA, Costa JC. Métodos laboratoriais para o diagnóstico da infecção pelo vírus da imunodeficiência humana (HIV). Medicina (Ribeirão Preto) 1999; 32:138-46.

13.Brasil. Ministério da Saúde. Secretaria de Projetos Especiais de Saúde. Coordenação Nacional de DST e AIDS. Análise dos resultados da primeira avaliação externa da qualidade (AEQ-1) para o diagnóstico sorológico da infecção pelo HIV-1 [online] 2000. Disponivel em URL: http://www.aids.gov.br

14. Shaffer N, Chuachoowong R, Mock PA, et al. Shortcourse zidovudine for perinatal HIV-1 transmission in Bangkok, Thailand: a randomised controlled trial. Lancet 1999; 353:773-80.

15.Administration of zidovudine during late pregnancy and delivery to prevent perinatal HIV transmission. Thailand, 1996-1998. MMWR Morb Mortal Wkly Rep 1998; 47:151-4.

16.Schramm W, Angulo GB, Torres PC, Burgess-Cassler A. A simple saliva-based test for detecting antibodies to human immunodeficiency virus. Clin Diagn Lab Immunol 1999; 6:577-80.

17.Kelen GD, Shahan JB, Quinn TC. Emergency department-based HIV screening and couseling: experience with rapid and standard serologic testing. Ann Emerg Med 1999; 33:147-55.

18.Lien TX, Tien NT, Chanpong GF, et al. Evaluation of rapid diagnostic tests for the detection of human immunodeficiency virus types 1 and 2, hepatitis B surface antigen, and syphilis in Ho Chi Minh City, Vietnam. Am J Trop Med Hyg 2000; 62:301-9.

19.Palmer CJ, Dubon JM, Koenig E, et al. Field evaluation of the Determine rapid human immunodeficiency virus diagnostic test in Honduras and the Dominican Republic. J Clin Microbiol 1999; 37:3698-700.

20.Arai H, Petchclai B, Khupulsup K, Kurimura T, Takeda K. Evaluation of a rapid immunochromatographic test for detection of antibodies to human immunodeficiency virus. J Clin Microbiol 1999; 37:367-70.

21.Stringer JS, Rouse DJ. Rapid testing and zidovudine treatment to prevent vertical transmission of human immunodeficiency virus in unregistered parturients: a cost-effectiveness analysis. Obstet Gynecol 1999; 94:34-40.

22.Duarte G, Mussi-Pinhata MM, Cervi MC, Kato CM, Paschoini SM, Quintana SM. Can isolated postpartum zidovudine prophylaxis reduce perinatal HIV transmission? Int J Gynecol Obstet 2000; 70 Suppl 1:123.

23.Grobman WA, Garcia PM. The cost-effectiveness of voluntary intrapartum rapid human immunodeficiency virus testing for women without adequate prenatal care. Am J Obstet Gynecol 1999; 181:1062-71. 